\title{
Editorial
}

\section{Iterative Methods and Applications}

\author{
Giuseppe Marino, ${ }^{1}$ Filomena Cianciaruso, ${ }^{1}$ Luigi Muglia, ${ }^{1}$ \\ Claudio H. Morales, ${ }^{2}$ and Daya Ram Sahu ${ }^{3}$ \\ ${ }^{1}$ Dipartimento di Matematica, Universitá della Calabria, 87036 Arcavacata di Rende, Italy \\ ${ }^{2}$ Department of Mathematics, University of Alabama in Huntsville, Huntsville, AL 35899, USA \\ ${ }^{3}$ Department of Mathematics, Banaras Hindu University, Varanasi 221005, India
}

Correspondence should be addressed to Giuseppe Marino; gmarino@unical.it

Received 10 December 2013; Accepted 10 December 2013; Published 9 March 2014

Copyright (c) 2014 Giuseppe Marino et al. This is an open access article distributed under the Creative Commons Attribution License, which permits unrestricted use, distribution, and reproduction in any medium, provided the original work is properly cited.

An important branch of nonlinear analysis theory, applied in the study of nonlinear phenomena in engineering, physics, and life sciences, is related to the existence of fixed points of nonlinear mappings, the approximation of fixed points of nonlinear operators, of zeros of nonlinear operators, and the approximation of solutions of variational inequalities.

This special issue is focused on the latest achievements in these topics and the related applications. The aim is to present the newest and extended coverage of the fundamental ideas, concepts, and important results of the topics below. Topics of interest include, but are not limited to the following.

(i) New iterative schemes to approximate fixed points of nonlinear mappings, common fixed points of nonlinear mappings, or semigroups of nonlinear mappings.

(ii) Iterative approximations of zeros of accretive-type operators.

(iii) Iterative approximations of solutions of variational inequalities problems or split feasibility problems and applications.

(iv) Optimization problems and their algorithmic approaches.

(v) Methods for the global continuation of fixed point curves in engineering problems.

(vi) Fixed point of nonlinear operators in cone metric spaces with applications and fixed points of nonlinear operators in ordered metric spaces with applications.
We invited the authors to present their original articles that will stimulate the continuing efforts in developing new results in the previous mentioned areas.

F. Zhang et al. introduce a new iterative scheme for finding a common fixed point of two countable families of multivalued quasinonexpansive mappings and prove a weak convergence theorem under the suitable control conditions in a uniformly convex Banach space.

S. M. Kang et al. establish the strong convergence for the hybrid S-iterative scheme associated with nonexpansive and Lipschitz strongly pseudocontractive mappings in Banach spaces.

R. Wangkeeree and P. Yimmuang first consider an auxiliary problem for the generalized mixed vector equilibrium problem with a relaxed monotone mapping and prove the existence and uniqueness of the solution for the auxiliary problem; then they introduce a new iterative scheme for approximating a common element of the set of solutions of a generalized mixed vector equilibrium problem with a relaxed monotone mapping and the set of common fixed points of a countable family of nonexpansive mappings.

Y. Tang and D. Wen prove strong convergence theorems for a common element of the set of fixed points of a finite family of pseudocontractive mappings and the set of solutions of a finite family of monotone mappings. The common element is the unique solution of a certain variational inequality.

L.-C. Ceng et al. introduce a new relaxed viscosity approximation method with regularization and prove the strong convergence of the method to a common fixed 
point of finitely many nonexpansive mappings and a strict pseudocontraction that also solves a convex minimization problem and a suitable equilibrium problem.

Recently, S. Takahashi and W. Takahashi proposed an iterative algorithm for finding common solutions of generalized equilibrium problems governed by inverse strongly monotone mappings and of fixed points problems for nonexpansive mappings.

H. Zhang and F. Wang provide a result that allows for the removal of one condition ensuring the strong convergence of the algorithm.

S. Cao introduces and analyzes the viscosity approximation algorithm for solving the split common fixed point problem for the strictly pseudononspreading mappings in Hilbert spaces.

Y. Wang presents a viscosity method for hierarchical fixed point problems to solve variational inequalities, where the involved mappings are nonexpansive nonself-mappings.

$\mathrm{H}$. Zegeye and $\mathrm{N}$. Shahzad introduce an iterative process which strongly converges to a common fixed point of finite family of uniformly continuous asymptotically strict pseudocontractive mappings in the intermediate sense.

C.-X. Li et al. present some comparison theorems for the spectral radius of double splittings of different matrices under suitable conditions, which are superior to the corresponding results in the recent papers.

P. Duan proposes an implicit iterative scheme and an explicit iterative scheme for finding a common element of the set of solutions of system of equilibrium problems and a constrained convex minimization problem by the general iterative methods in Hilbert spaces.

H. Sun and Z. Xue demonstrate that the proof of main theorem of B. E. Rhoades and Stefan M. Soltuz in J. Math. Anal. Appl. 283 (2003), 681-688, is incorrect. The authors provide the correct version of this result concerning the equivalence between the convergences of Ishikawa and Mann iterations for uniformly L-Lipschitzian asymptotically pseudo-contractive maps. S. M. Kang et al. study the convergence of implicit Picard iterative sequences for strongly accretive and strongly pseudocontractive mappings.

G. Chiaselotti et al. use a discrete dynamical model with three evolution rules in order to analyze the structure of a partially ordered set of signed integer partitions whose main properties are actually not known. This model is related to the study of some extremal combinatorial sum problems.

Applying the Taylor and multiplication rule of two generalized polynomials, H.-K. Liu develops a series solution of linear homogeneous q-difference equations.

$\mathrm{X}$. Mu and Y. Zhang, among the others, present a ranktwo feasible direction algorithm based on the semide nite programming relaxation of the binary quadratic programming. The proposed algorithm restricts the rank of matrix variable to be two in the semide nite programming relaxation, and yields a quadratic objective function with simple quadratic constraints. Moreover a feasible direction algorithm is used to solve the nonlinear programming and the convergent analysis and time complexity of the method is given.
M. De la Sen investigates the limit properties of distances and the existence and uniqueness of fixed points, best proximity points, and existence and uniqueness of limit cycles, to which the iterated sequences converge, of single-valued, and so-called, contractive precyclic self-mappings.

X.-F. Zhang et al. propose three kinds of preconditioners to accelerate the generalized AOR (GAOR) method for the linear system from the generalized least squares problem. The convergence and comparison results are also obtained.

L. Bergamaschi and A. Martínez propose a parallel preconditioner for the Newton method in the computation of the leftmost eigenpairs of large and sparse symmetric positive definite matrices.

J. Park et al. apply a numerical procedure introduced by Choi and Jang in 2012 for the numerical analyzing of static defection of an infinite beam on a nonlinear elastic foundation.

G. Chiaselotti et al. introduce the concept of fundamental sequence for a finite graded poset $\mathrm{X}$ which is also a discrete dynamical model.

J. Iqbal and M. Arif study symmetric successive overrelaxation (SSOR) method for absolute complementarity problems.

\section{Acknowledgment}

The editors would like to thank the authors for their interesting contributions.

Giuseppe Marino
Filomena Cianciaruso
Luigi Muglia
Claudio H. Morales
Daya Ram Sahu




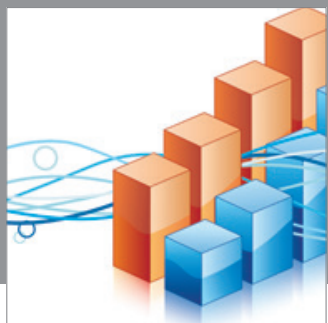

Advances in

Operations Research

mansans

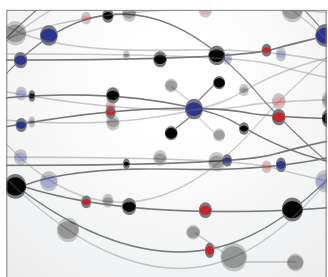

The Scientific World Journal
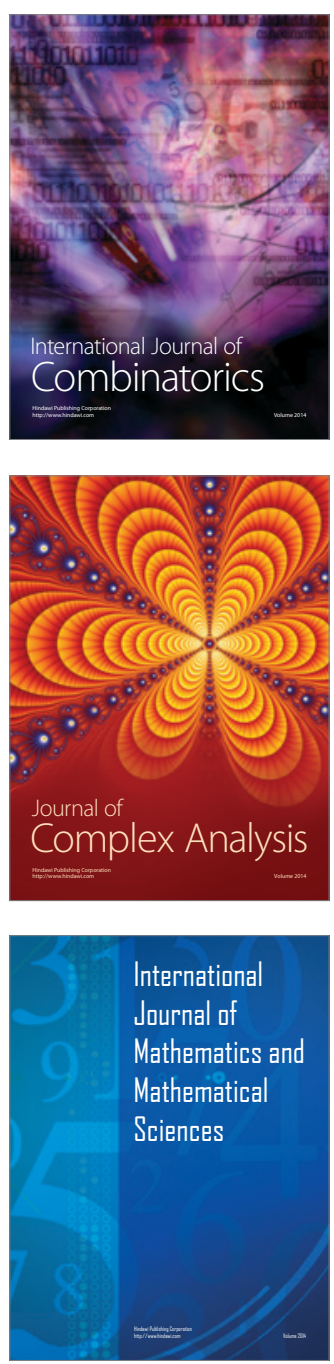
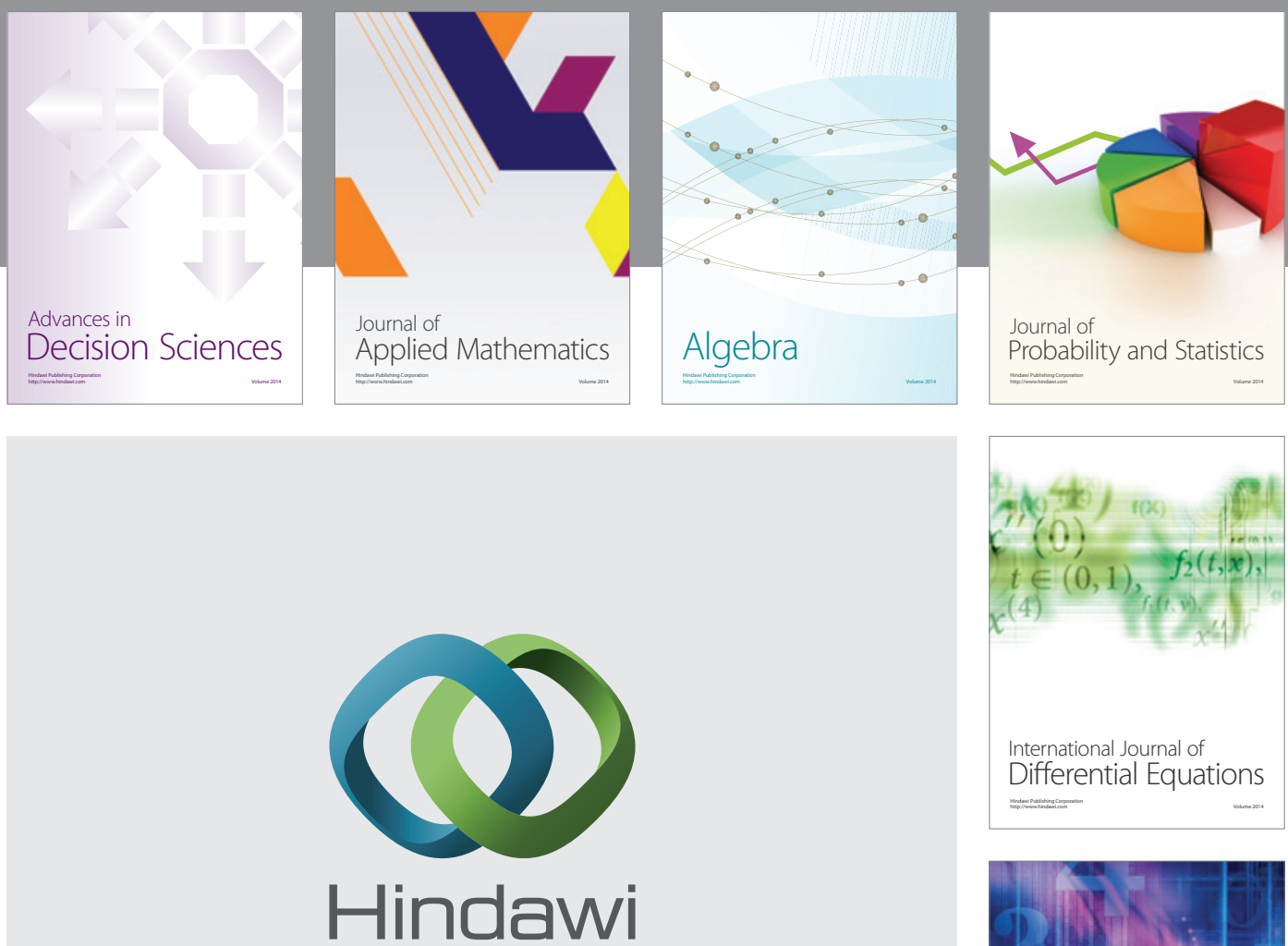

Submit your manuscripts at http://www.hindawi.com
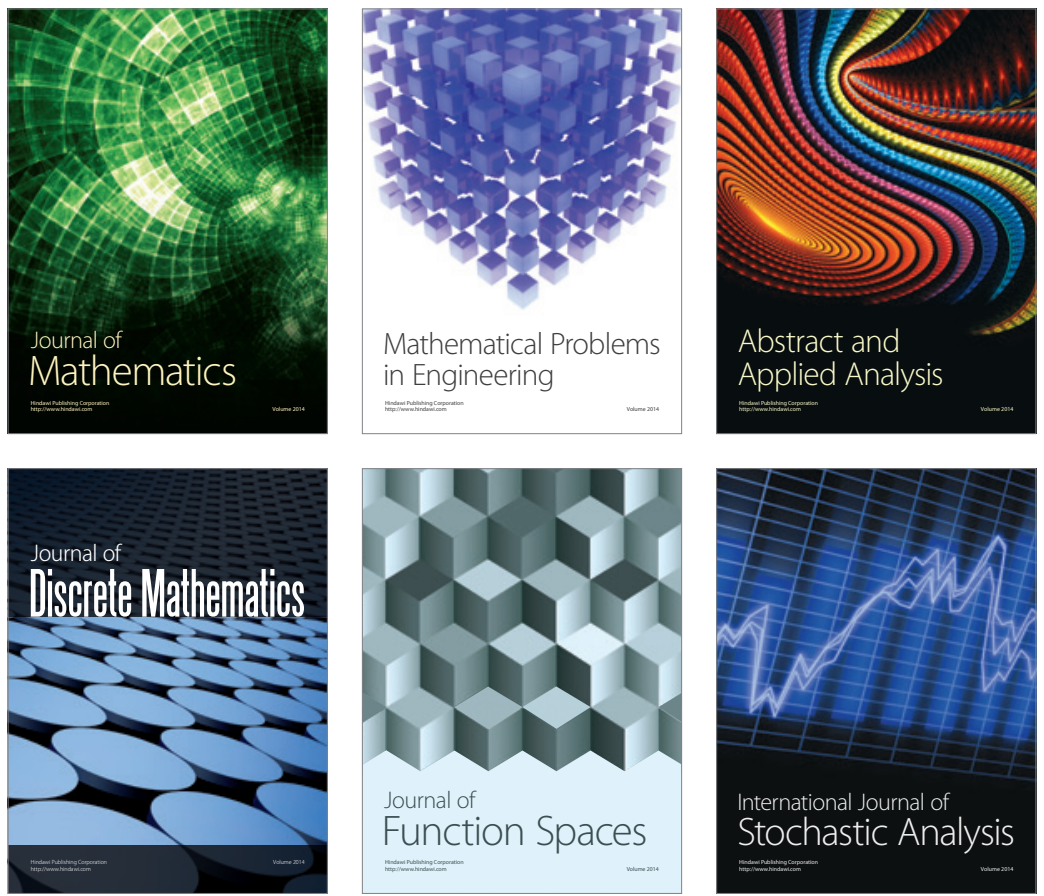

Journal of

Function Spaces

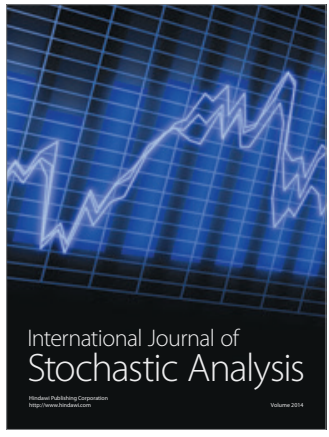

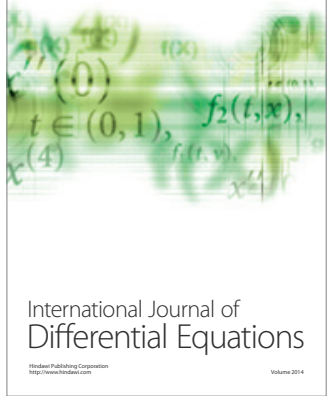
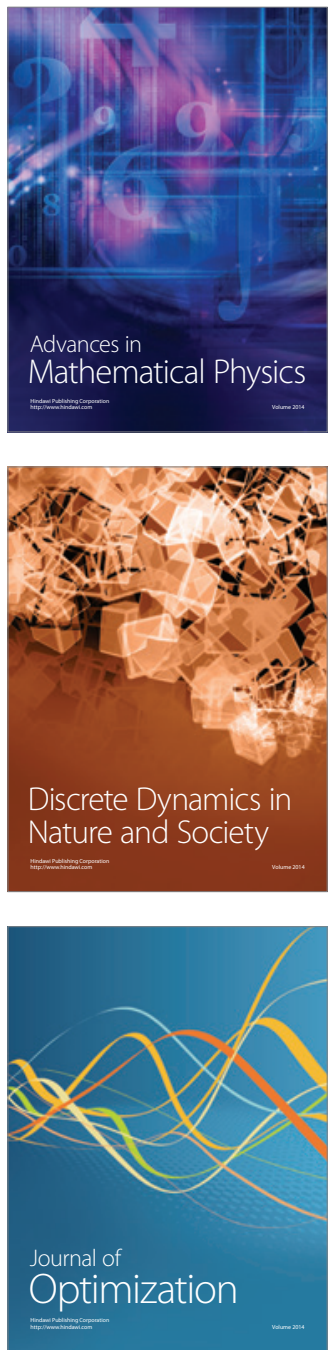